\section{Nephrocytes: a role} in immunity

Peptidoglycans (PGNs) are pathogenassociated molecular patterns (PAMPs) that are shed by bacteria, including those of the microbiome, to stimulate an immune response. Uncontrolled immune stimulation can lead to chronic inflammation and shortened lifespan; hence, mechanisms exist to prevent excessive immune activation in response to PAMPs. New research in Drosophila demonstrates a role for nephrocytes in this regulatory process. "We show that nephrocytes remove microbiota-derived PGN fragments from the circulation to prevent aberrant immune activation," explains Nicolas Buchon. "Thus, renal filtration of microbiota-derived PGN maintains immune homeostasis in Drosophila."

Drosophila nephrocytes have molecular and functional similarities to podocytes and to cells of the reticuloendothelial system. To assess the role of nephrocytes and haemolymph (insect blood) filtration in immunity, Buchon and colleagues studied Drosophila with a mutation in Klf15 - the ortholog of human KLF15, which is required for podocyte differentiation - which lack nephrocytes. Despite the known ability of nephrocytes to detoxify hemolymph, Buchon and colleagues found that Klf15-null flies were more resistant to infection than wild-type flies. Further analyses showed that nephrocytes of wild-type flies removed PGN from the circulation via endocytosis and subsequent lysosomal degradation. This process was impaired in Klf15-null flies, resulting in excess PGN in haemolymph and constitutive activation of the Toll pathway. Despite their resistance to infection, Klf15-null flies had shorter lifespans than wild-type flies. This difference was, however, abolished by raising the flies under germ-free conditions, suggesting that the shortened lifespan of conventionally housed Klf15-null flies resulted from an aberrant immune response to microbiota.

The researchers propose that renal or reticuloendothelial systems could have similar immunoregulatory functions in mammals. "Renal filtration could regulate the levels of microbiota-derived PAMPs, such as PGN, in the blood, to maintain immune homeostasis," says Buchon. "Thus impairment of renal function could directly induce chronic immune activation with proinflammatory effects."

Susan J. Allison

ORIGINAL ARTICLE Troha, K. et al. Nephrocytes remove microbiota-derived peptidoglycan from systemic circulation to maintain immune homeostasis. Immunity https://doi.org/ 10.1016/j.immuni.2019.08.020 (2019)

\title{
Uromodulin regulates oxidative stress
}

A new study reports that the kidney-derived protein uromodulin is a regulator of oxidative stress. Although the majority of uromodulin is secreted into the urine, a small amount is released into the kidney interstitium and circulation.

"Our longstanding hypothesis is that uromodulin is a defender of kidney and systemic homeostasis," explains researcher Tarek El-Achkar. "Supporting this hypothesis, recent studies have shown that high levels of circulating uromodulin are associated with reduced mortality and reduced risk of chronic kidney disease (CKD) and acute kidney injury (AKI)."

To investigate the molecular signals that are regulated by uromodulin in kidney cells, El-Achkar and colleagues used unbiased omics approaches. They show that uromodulin inhibits RAC1-JNK activation in proximal tubule cells and that uromodulin deficiency leads to increased systemic and renal oxidative stress.

In a mouse model and in patients undergoing liver transplantation, AKI resulted in a reduction in serum uromodulin levels and an increase in systemic oxidative DNA damage. Furthermore, high levels of oxidative DNA damage were associated with mortality and need for dialysis in the patients who developed AKI.

Finally, the researchers show that uromodulin inhibits activation of the nonselective calcium ion channel TRPM2, which has previously been shown to have a role in RAC1-dependent signalling and oxidative stress in AKI. They demonstrate that the increase in systemic oxidative stress that is induced by uromodulin deficiency is TRPM2-dependent.

"Regulation of oxidative stress could explain the link between serum uromodulin levels and systemic outcomes," says El-Achkar. "If our findings are validated in large human studies, we envision future therapeutic strategies that aim to increase the levels of systemic uromodulin in specific clinical situations characterized by profound uromodulin deficiency."

Ellen F. Carney

ORIGINAL ARTICLE LaFavers, K. A. et al. Circulating uromodulin inhibits systemic oxidative stress by inactivating the TRPM2 channel. Sci. Transl Med. 11, eaaw3639 (2019)

\section{Synthetic lethality between loss of CDK 4/ 6 activity and VHL inactivation}

Nearly all cases of clear cell renal cell carcinoma (ccRCC) are associated with inactivating mutations or hypermethylation of the von Hippel-Lindau tumour suppressor gene VHL. The resultant dysfunctional $\mathrm{pVHL}$ leads to accumulation of hypoxia-inducible factor $2 \alpha$ (HIF2a), which activates proliferative and angiogenic pathways. Although small-molecule inhibitors of HIF2 $\alpha$ have shown some promise for the treatment of ccRCC, the response can be variable. The identification of synthetic lethality between decreased activity of cyclindependent kinases 4 and 6 (CDK4/6) and VHL inactivation in human and Drosophila ccRCC cell lines suggests that the addition of a CDK4/6 inhibitor to a HIF2 $\alpha$ inhibitor might improve outcomes for patients with ccRCC.

Synthetic lethality occurs when a deficiency in one gene is tolerated, whereas a combination of deficiencies in genes leads to cell death. The identification of targets that have synthetic lethal relationships with genes that are mutated in cancer is an appealing therapeutic approach to specifically target tumour cells. To identify targets with synthetic lethal relationships with VHL loss, Nicholson and colleagues performed synthetic lethal screens in Drosophila cells using RNA interference and human ccRCC cells using a library of chemicals with known anticancer activity. These screens led to the identification of CDK $4 / 6$ as being synthetic lethal with loss of $\mathrm{VHL}$. This synthetic lethal relationship was independent of HIF $2 \alpha$; thus, the antiproliferative effects of CDK4/6 inhibition were synergistic with HIF2a inhibition in VHL-defective ccRCC cells that were HIF2 $\alpha$ dependent, while still attenuating proliferation of VHL-defective ccRCC cells that were HIF2 $\alpha$ independent. CDK4/6 inhibition also inhibited the growth of ccRCC orthotopic xenografts in mice regardless of HIF2 $a$ dependency.

Susan J. Allison

ORIGINAL ARTICLE Nicholson, H. E. et al. HIF-independent synthetic lethality between CDK $4 / 6$ inhibition and VHL loss across species. Sci. Signal. 12, eaay0482 (2019) 